\title{
Rett Syndrome
}

\author{
E.E.J. Smeets ${ }^{a} \quad$ K. Pelc ${ }^{b} \quad$ B. Dan ${ }^{b}$ \\ ${ }^{a}$ Department of Clinical Genetics, Maastricht University Medical Center, Maastricht, The Netherlands; \\ ${ }^{b}$ Department of Neurology, Hôpital Universitaire des Enfants Reine Fabiola, Université Libre de Bruxelles (ULB), \\ Brussels, Belgium
}

\section{Key Words}

$\mathrm{MeCP} 2 \cdot$ Rett syndrome

\begin{abstract}
Rett syndrome is one of the most common causes of complex disability in girls. It is characterized by early neurological regression that severely affects motor, cognitive and communication skills, by autonomic dysfunction and often a seizure disorder. It is a monogenic X-linked dominant neurodevelopmental disorder related to mutation in $M E C P 2$, which encodes the methyl-CpG-binding protein MeCP2. There are several mouse models either based on conditional knocking out of the Mecp2 gene or on a truncating mutation. We discuss the clinical aspects with special emphasis on the behavioral phenotype and we review current perspectives in clinical management alongside with perspectives in altering gene expression.

Copyright $\odot 2012$ S. Karger AG, Basel
\end{abstract}

\section{History}

In 1954, Andreas Rett, a pediatric neurologist in Vienna, first recognized the characteristic features of the syndrome which later came to bear his name. His publication in the German medical literature in 1966 [Rett, 1966], however, remained largely unnoticed. In the large textbook series on Neurology by Vincken and Bruyn, A. Rett wrote a chapter under the misleading heading of
'Cerebral Atrophy and Hyperammonaemia' in a series of 21 girls and women [Rett, 1977]. Other child neurologists observed the same clinical presentation in Japanese girls [Ishikawa et al., 1978]. It was, however, Bengt Hagberg from Gothenburg who shared his clinical observations in a Swedish survey at the Manchester Meeting on Child Neurology in 1981 and revealed this unique syndrome to the international medical world in 1983 [Hagberg et al., 1983]. Very soon a well-organized parental support group started and the International Rett Syndrome Association has now become one of the largest research and parent support foundations in the world, continuously encouraging the medical researchers and clinicians to seek for keys to unlock the biological riddle of this disorder. Meanwhile, parents all over the world have organized their continental and national parental support and advocacy groups. Collaboration between physicians and such groups has contributed to the improvement of knowledge by research, developing appropriate information for patients, health professionals and the general public, and promoting access to screening and diagnostic testing as well as to quality treatment and social benefits [Dan, 2008].

Rett syndrome (RTT) is a clinical diagnosis based on internationally accepted diagnostic criteria that were developed and adapted over the years as a useful tool for the clinicians who are involved in the diagnostic work-up and for researchers in RTT-related science [Hagberg et al., 2002; Neul et al., 2010]. It is considered to be one of the most common causes of complex disability in girls. In the

\section{KARGER}

Fax +4161306 1234

E-Mail karger@karger.ch

www.karger.com
(C) 2012 S. Karger AG, Basel

$1661-8769 / 11 / 0025-0113 \$ 38.00 / 0$

Accessible online at:

www.karger.com/msy
Eric E. J. Smeets

Department of Clinical Genetics

Maastricht University Medical Center

PO Box 5800, NL-6202 AZ Maastricht (The Netherlands)

Tel. +31 43387 5778, E-Mail eric.smeets@mumc.nl 

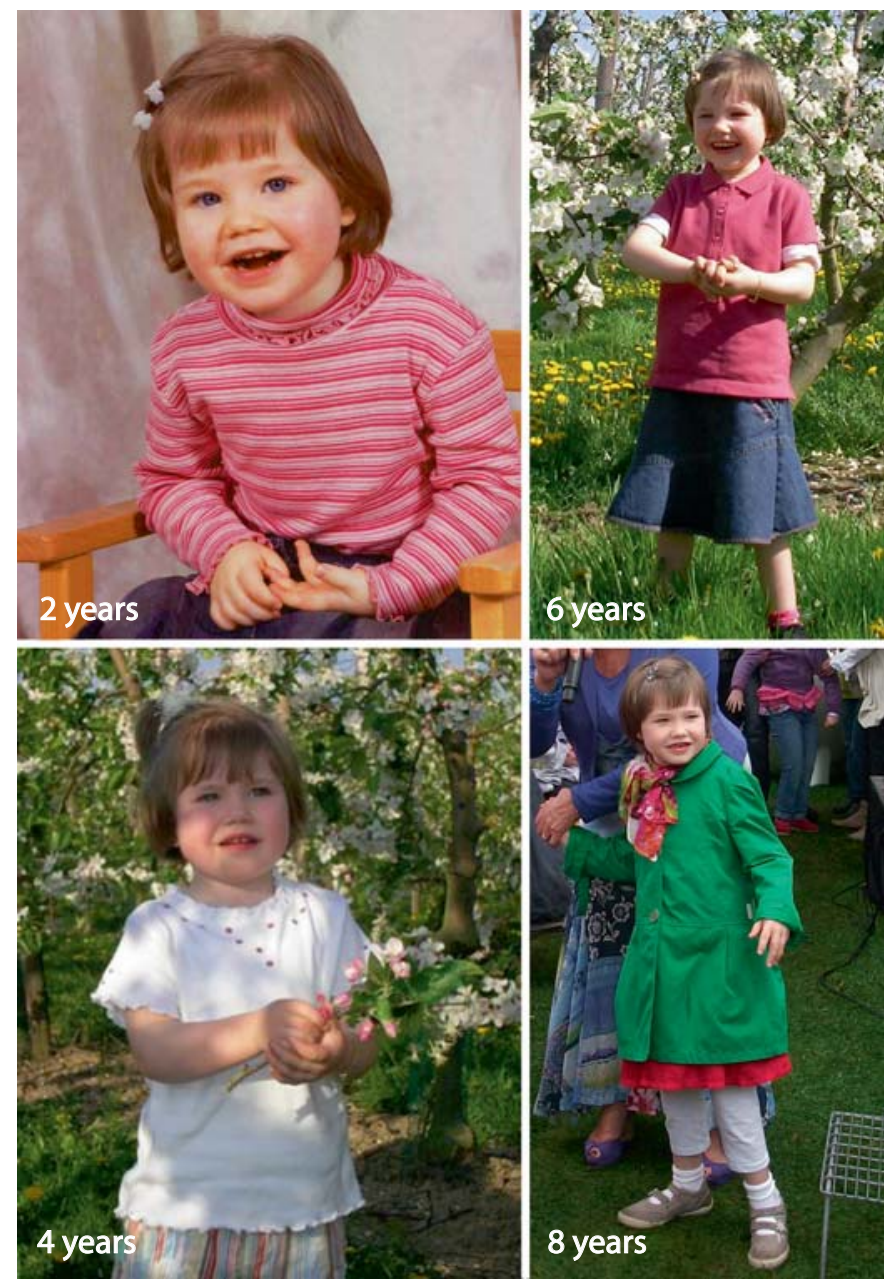

Fig. 1. Series of photographs of a 9-year-old girl with RTT diagnosed at 2 years, forceful breather at 6 years, very sociable at 8 years.

late 1990s, researchers from Huda Zoghbi's laboratory suggested that the MECP2 gene, located on the X chromosome, was a good candidate gene for RTT based on some unexpected experimental results from an unrelated project and then demonstrated MECP2 mutations in a number of patients with RTT [Amir et al., 1999]. RTT was thus the first neurodevelopmental disorder related to a defective transcription of methylated DNA. Some of the variant phenotypes initially thought to be very similar to RTT are now known to be caused by mutations in other genes. The congenital variant of RTT is related to FOXG1 and the infantile seizure onset variant (Hanefeld variant) is related to CDKL5. Others disorders with overbreathing include the Pitt-Hopkins syndrome, related to TCF4 haploinsufficiency and the CNTNAP2- and NRXN1-related
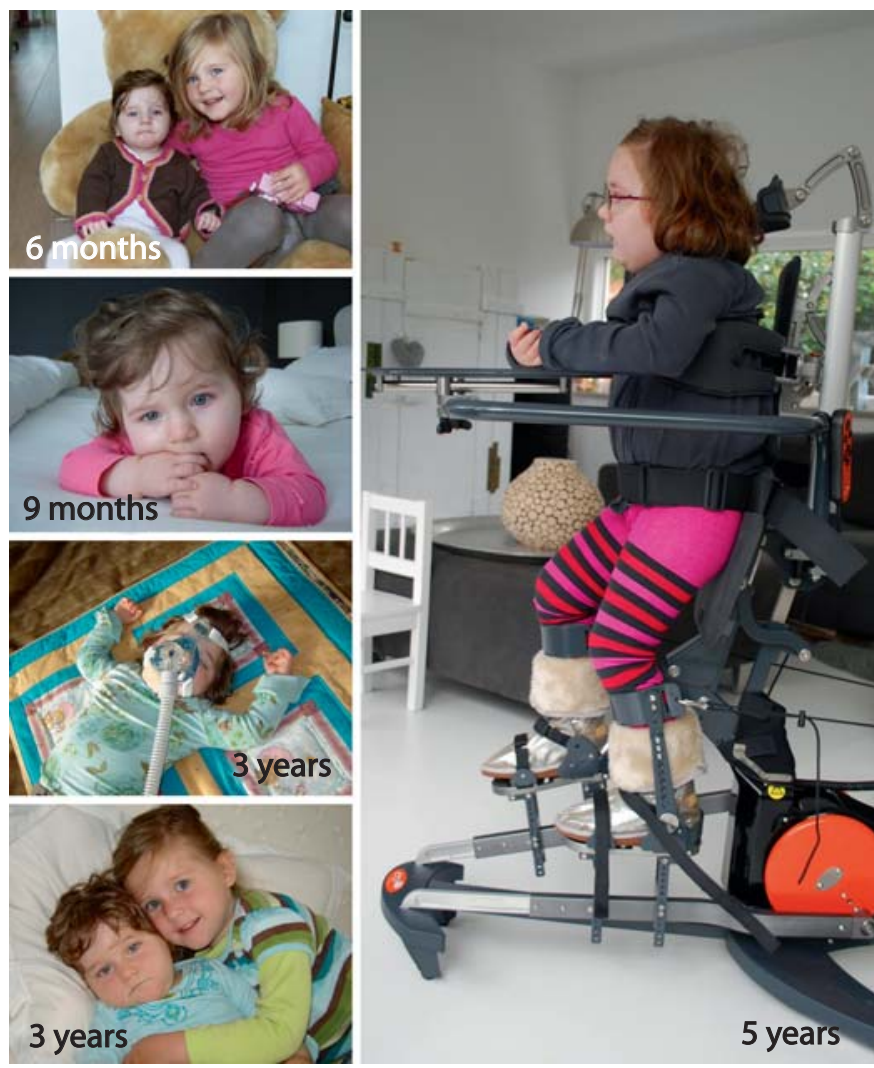

Fig. 2. Series of photographs of an 11.5-year-old girl with RTT. She is a feeble breather: notice the absent gaze at 9 months, BIPAP ventilation during sleep at 3 years and alert behavior during daytime at 5 years.

disorders with severe intellectual disability, autism and breathing abnormalities resembling Pitt-Hopkins syndrome [Zweier et al., 2009].

The prevalence of the syndrome in males with a normal karyotype and no family history of an affected female is certainly very low. For this purpose, here, only the female phenotype will be described.

\section{Clinical Features}

RTT is characterized by early neurological regression that severely affects motor, cognitive and communication skills, often leading to microcephaly, a delay in acquiring new skills, absence of speech, emergence of autistic features, loss of purposeful manipulation skills, replaced by stereotyped hand movements, other motor abnormalities including abnormal muscle tone, ataxia and apraxia, and often a seizure disorder (fig. 1,2). The clinical features 
Table 1. Rett syndrome diagnostic criteria 2010 [Neul et al., 2010]

Consider RTT diagnosis when postnatal deceleration of head growth is observed

Required for typical or classic RTT

A period of regression followed by recovery or stabilization

1. All main and all exclusive criteria

2. Supportive criteria are not required, although often present in typical RTT

Required for atypical or variant RTT

1. A period of regression followed by recovery or stabilization

2. At least 2 of the 4 main criteria

3. 5 out of 11 supportive criteria

Main criteria

1. Partial or complete loss of acquired purposeful hand skills

2. Partial or complete loss of acquired spoken language

3. Gait abnormalities: impaired (dyspraxia) or absence of ability (apraxia)

4. Stereotypic hand movements such as hand wringing/ squeezing, clapping/tapping, mouthing and washing/ rubbing automatisms

Exclusion criteria for typical RTT

1. Brain injury secondary to trauma (peri- or postnatally), neurometabolic disease or severe infection that cause neurological problems

2. Grossly abnormal psychomotor development in the first 6 months of life

Supportive criteria for atypical RTT

1. Breathing disturbances when awake

2. Bruxism when awake

3. Impaired sleep pattern

4. Abnormal muscle tone

5. Peripheral vasomotor disturbances

6. Scoliosis/kyphosis

7. Growth retardation

8. Small cold hands and feet

9. Inappropriate laughing/screaming spells

10. Diminished sensitivity to pain

11. Intense eye communication and eye-pointing behavior

form the basis for the clinical diagnosis (table 1). Many girls with RTT have autonomic perturbations, including irregular breathing. This includes hypoventilation or hyperventilation during wakefulness, breath-holding, aerophagia, forced expulsion of air and saliva, and apnea.

An infant girl developing RTT often has a strange and puzzling behavior. Retrospective analysis of home videos shows that hypotonia is usually present before 6 months of age; general developmental progress may be suboptimal during the first year; especially the developing eyehand coordination patterns are recorded as peculiar and inadequate. But the overall developmental pattern is not obviously disturbed in the first year. Then the character- istic features of RTT appear successively. Currently, RTT is no longer considered as progressive neurodegeneration but as a neurodevelopmental disorder.

\section{The RTT Cascade of Clinical Symptoms in a Staging System}

The cascade of clinical symptoms with evolving communicative dysfunction and loss of acquired skills and motor performance was delineated in a staging system [Witt Engerström, 1990; Hagberg and Gillberg, 1993] designed to help clinicians to discriminate between the very non-specific developmental profile early in life and the more specific profile for the disorder in later life. The 4 clinical stages are described below.

The Early-Onset Stagnation Period (Stage I). This occurs between 6 months and 1.5 years of age. A more or less sudden change in the interactive behavior of the baby girl is noted by the parents, especially by the mother (she is almost always the first to become aware about a change in contact with her baby). Her daughter seems to demand little attention from her. Others may be irritable and restless and parents may relate frequent crying to teething. Postural development is still progressing but at a delayed rate [Dan and Cheron, 2008]. She may learn to sit upright but not to crawl or to stand up and bottom-shuffling is very common. Babbling and new words appear but remain poor. Since the overall developmental pattern of their child is still grossly normal, parents are usually reassured by the first-line medical practitioners.

The Rapid Developmental Regression Period (Stage II). Transition to this stage occurs between 1 and 4 years of age and is characterized by rapid and specific regression of acquired abilities. This happens often very suddenly, sometimes even with pseudo-toxic symptoms (highpitched crying, fever and apathy suggesting meningoencephalitis) leading to emergency hospitalization. The pediatrician is left without clinical explanation because there are no abnormal biochemical or microbiological findings. Such an acute episode can last for days or weeks. After recovery, the child's personality has changed completely. Other affected children demonstrate a more gradual decline in their communicative ability and motor performance. Although eye contact is preserved, they show diminished interest in people and objects. Previously acquired babbling, words and fine motor skills are lost during this period and mental deficiency now becomes obvious. The exploratory character of her play becomes poor or is lost. Grasping or reaching out for objects and toys ends abruptly in withdrawal and in senseless hair-pulling or head-tapping behavior. Later it will pro- 
ceed to peculiar posturing of wrists and twisting of the fingers. During this phase, parents feel that they lost contact with their daughter as her visual contact has changed and she appears to be absent. Crying at night, recurrent infections or bouts of unexplained fever are common in this stage and often accepted as an explanation for the delay in development. Febrile seizures may be present and questions about epileptic paroxysms may arise. Unremarked panting, spitting, hypersalivation and hyperventilation, together with muscle twitching around the corners of the mouth and facial grimacing, are now considered to be the emerging manifestations of 'brainstem immaturity'. Decline in head growth is noticed in many but not all of them.

The Pseudo-Stationary Stage (Stage III). This stage starts when the regression stage is over. If walking was previously acquired, the child may still be able to do so. Others may even still learn this skill. But the loss of purposeful hand use is obvious. The typical hand stereotypies now become prominent and constitute the hallmark of the disorder. They start as continuous repetitive midline movements with hand wringing, hand washing and clapping as soon as the child is awake. Others display hand stereotypies along their bodies. The fingers are twisted in unusual ways. The visual contact behavior returns suggesting an 'awakening' and some of the former personality of the child is recognized again by her parents. She is now more alert and joyful with typical eye-pointing behavior to express needs and wishes. Breathing irregularities, although modestly present in stage II, may become more prominent in this stage, with or without non-epileptic vacant spells, apneustic or feeble breathing, hyperventilation and Valsalva maneuver-type of breathing. Unexplained night laughing, frequent daytime sleeping and nighttime awakening, crying spells and sudden agitation occur. Many have overt clinical epilepsy requiring treatment, but many also become seizure-free after some time. Dystonic asymmetrical posture leads to neurogenic scoliosis, often rapidly progressive, requiring surgical treatment. The feet and lower limbs are cold, with or without color change, and with or without atrophic changes. Shortening of Achilles tendons in equinus position causes deformation of the ankles into varus or valgus position. The toes are held typically clenched. Some girls with milder phenotype have preserved speech and/or hand use. Motor regression slowly progresses in this stage, in contrast to a remarkably well-preserved ability to communicate mainly with the eyes. In this stage, that can last for decades, the girls and women still learn effectively about new things, situations and persons.
Late Motor Deterioration (Stage IVA). This stage starts when walking ceases and the individual with RTT becomes wheelchair-dependent. Some individuals with severe manifestations never learn to walk and pass immediately from stage II into stage IVB, which is characterized by severe neurological impairment with pronounced muscle wasting and distortion of distal limbs. The feet are cold and discolored with abiotrophic changes. Hand stereotypies become less intense and simpler with age. Lack of motor activity in these quadriplegic women finally leads to a state of frozen rigidity. However, remarkable visual contact and eye-pointing behavior remain present even in the most severe situation and it is crucial to recognize it to foster communication and participation.

\section{Diagnostic Criteria}

In spite of molecular genetics RTT remains a clinical diagnosis. It rests on diagnostic criteria that were reappraised taking into account cumulative advances in our understanding of the clinical, neurobiological and molecular genetic characteristics of the syndrome (table 1) [Hagberg et al., 2002; Neul et al., 2010]. Diagnostic criteria have also been suggested for variant phenotypes (table 2) which meet at least 2 of 4 main criteria (a clinical profile characterized by a regression stage followed by a recovery of interaction while neuromotor regression continues, reduced hand skills, hand stereotypies, reduced babble speech, reduced communication skills) and at least 5 of 11 supportive criteria (breathing irregularities, air swallowing, bruxism, kypho-scoliosis, abnormal gait, lower limb amyotrophy, cold, purplish feet, diminished response to pain, sleep disturbances, laughing/screaming spells and intense eye contact).

It is stated that for the diagnosis of atypical RTT an individual must have a period of regression followed by recovery or stabilization. In addition to having a regression, individuals must have at least 2 of the 4 main criteria and 5 of 11 supportive criteria. This clearly distinguishes these cases not only from relentless degenerative disorders but also from the congenital and infantile seizure onset RTT variant that is related to mutations in FOXG1 and CDKL5, respectively. These disorders are now accepted as separate entities, different from MECP2-related RTT.

\section{Features Common in RTT}

Feeding and Physical Growth. Girls with RTT love to eat. They like to watch when meals are prepared and are very alert during feeding. Emergence and control of primary mouth functions such as chewing and swallowing 
Table 2. The cardinal clinical features of Rett syndrome in relation to pathology [Julu et al., 2008]

\begin{tabular}{lll}
\hline Affected part & Reported pathology & Clinical observations \\
\hline Cortical & $\begin{array}{l}\text { Decreased dendritic arborization and smaller } \\
\text { than normal brain }\end{array}$ & Severe intellectual disability \\
\hline Cortical & Epilepsy & Seizures \\
\hline Extrapyramidal & Monoaminergic dysfunction & $\begin{array}{l}\text { Dystonia, incoordination of motor activities, secondary orthopedic } \\
\text { deformities and muscle wasting with contractures }\end{array}$ \\
\hline Brainstem & Monoaminergic dysfunction & Dyspraxia, agitation and sleep disturbances \\
\hline Brainstem & $\begin{array}{l}\text { Immaturity with incompetence of inhibitory } \\
\text { neuronal networks }\end{array}$ & $\begin{array}{l}\text { Abnormal breathing rhythms and lack of integrative inhibitions } \\
\text { are likely causes of sudden deaths }\end{array}$ \\
\hline Brainstem & Dysautonomia & \begin{tabular}{l} 
Cold and blue extremities and sympatho-vagal imbalance \\
\hline
\end{tabular}
\end{tabular}

are often delayed and problematic. Most of the girls have reduced movements of the mid- and posterior tongue, with premature spill-over of food and liquid from the mouth into the pharynx [Motil et al., 1999]. They also show delayed pharyngeal swallowing, but otherwise pharyngeal problems are minimal. There is a high incidence of gastroesophageal reflux and decreased intestinal motility resulting in constipation. Because of the Valsalva breathing pattern and pressing the air over a closed glottis, air swallowing is common. The resultant bloating of the abdomen can be extreme and reach a degree that is not encountered in other disorders with intellectual disability. These clinical features may interfere with normal feeding and with general comfort. The rate of hand and foot growth, particularly the latter, of girls with RTT is slower than that of the normal female [Schultz et al., 1998].

There is deceleration of linear growth during the first 2 years of life. Later on, height and/or weight for height often fall 2 SD below normal. Daily energy and water requirements can be much higher than is often realized, particularly in forceful breathers or when Valsalva-maneuver type of breathing is frequent. It must be noted that whereas Valsalva is present in all RTT phenotypes, including in patients with apneustic or feeble breathing, though it is more readily noted in forceful breathers (i.e. with marked hyperventilation). The mean head circumference in classic RTT tends to fall 2 SD below the norm by the age of 4 years. After the age of 8 years, it stabilizes close to 3 SD below the mean. Head growth has decelerated by less than 1 SD in $20 \%$ of the girls at the age of 6 years and in $10 \%$ at an age of 12 years. In 'forme fruste' variants, head circumference remains within or close to normal limits, though head size is $0.8 \mathrm{SD}$ below average
[Hagberg et al., 2001]. The decline in head growth may thus be very obvious in classical RTT, but it may not be present at all in atypical RTT. Therefore, microcephaly is no longer an essential criterion for RTT diagnosis.

Communication. All girls with RTT develop intellectual disability to a variable extent. The absence of speech in most affected girls, the dyspraxia and the short attention span with lack of interest in play makes developmental testing a difficult task. Affected children try to establish visual contact by intense staring. They look at you and want to be looked at. Therefore, particular care should be taken to preserve visual sensory function. No specific ophthalmologic pathology occurs in this disorder, although strabismus is very common. Acquired cataracts may occur after self-injurious tapping in association with behavioral agitation. Retinal changes are not present. In the long-lasting stage III of 'wake-up' and 'pseudostabilization', parents become experts in understanding and recognizing the wishes of their daughter. They 'speak' to each other 'through' the eyes. This intense eye contact behavior is further accentuated in older affected females in a typical eye-pointing behavior which expresses wishes and remains present even in the most severely affected female. Teachers can use this eye-pinpointing behavior to develop alternative communication in habilitation programs. This intense eye contact and eye-pointing behavior is very distinctive of RTT compared to other severe intellectual disability and/or autistic conditions. Girls with RTT are able to make choices and take causally-related action. But they need more time taking into account certain latency in producing the response. Therefore, their parents and caregivers should be aware that the time they require to show what is wanted or to produce their response to a specific situation is prolonged. Some girls 
with RTT have preservation of speech or use words and sentences in a meaningful way. Some of them continue to learn new words and names far into stage III and into adulthood. Gradual loss of this speech ability usually may coincide with the time when walking stops, i.e. transition into stage IV. It is the overall impression of parents that their daughters actually understand more of the ongoing conversation than is generally considered possible. Their comprehension is probably largely situation-based or related to the facial expression and emotional attitude of the adult. Girls and adults in stage III display a happy demeanor and gently feel people with hands and feet in playful body contact. Others may reject physical touch of particular body parts and get agitated when they feel unsafe. Their preference for male caretakers or visitors is well-known and will be confirmed by many parents. Autistic features predominate in some of the females with variant MeCP2-related RTT. Odd behaviors, attention deficit and hyperactivity are common, but careful observation will indicate association with subtle change in muscle tone, including dystonic posture.

Hand Stereotypies. They are the hallmark of this disorder. The stereotyped movements are typically of small magnitude, but they may be accompanied by more proximal movements, that may also involve the trunk [Dan and Cheron, 2008]. They include unimanual or bimanual wringing, mouthing, rubbing, patting, squeezing and clapping. They change with emotional state and are often associated either directly or inversely with respiratory patterns [Elian and de M Rudolf, 1996]. They tend to appear when the patient is sitting, standing or walking. These stereotypies disturb communicative interaction through distraction and agitation.

Kyphoscoliosis. Mild trunk hypotonia and lack of mobility often lead to kyphoscoliosis. Scoliosis develops in early school age with various degrees of severity. Sometimes progression is very rapid, depending on asymmetry in muscle tone (dystonia) and the degree of muscle wasting. In ambulatory girls, scoliosis appears unpredictable. It may never be present or may only develop to a small extent. In non-ambulatory girls with classical RTT stage IVB scoliosis develops in spite of preventive measures. Most commonly an S-shaped curve develops with a longer upper part (most frequently dextroconvex) and a shorter lower part (sinistroconvex). It should be stressed that progression of spine deviation continues even in adulthood. Kyphosis occurs more frequently in ambulatory girls. Tiptoe walking in girls with RTT, in contrast to other circumstances with neurologic deficit, seems to be related to uncertainty and anxiety about falling. Girls gain support and stability by bending forward on stiff legs, giving them more balance against gravity. While sitting and drowsy, girls tend to drop their heads forward causing more bending of the cervical and high thoracic spine.

Foot Deformities. Reduced intersegmental mobility, related to stereotyped postures or to dystonia in the context of an extrapyramidal syndrome and, to a lesser extent, to a pyramidal syndrome, may lead to joint deformity. Most common foot deformities seen in RTT are equinus and equinovalgus/varus positions. Shortening of the Achilles tendons is then compensated for by an 'escape' in the valgus or varus position. Young girls do not suffer from this and continue to develop walking ability. If there is hyperextension of the ankles, the need for compensation rises to the knees, the hips and the spine, threatening loss of balance and making walking very difficult if not impossible.

Peculiar Gait. From the 3rd or 4th year, gait is characterized by ataxia, apraxia and, in some girls, dynamic equinus [Dan and Cheron, 2008]. Two distinct patterns of ground contact have been described, namely plantigrade gait with continuous heel contact throughout the stance phase and digitigrade gait, making ground contact with the first or the fifth metatarsal without contact of the heel. The plantigrade pattern is further characterized by a lack of limb-girdle dissociation, excessive lateral movement of the trunk and wide base. The digitigrade pattern is associated with relatively good proximal selectivity of movements, contrasting with patterns seen in spastic diplegia or paraparesis. Affected girls develop a preference for 1 leg, putting it forward at every step as the foremost leg, using the other leg as support and balance. Direction is chosen through the foremost leg. Sometimes the other leg is placed more to the side causing the girl to walk in circles. Predominantly distal asynchronous 6-8 bursts of electromyographic activity per second are recorded during gait and might contribute to abnormal control [Pelc and Dan, 2009].

Seizures. Epilepsy is present in up to $80 \%$ of affected individuals at some time in their lives [Steffenburg et al., 2001]. It usually starts after the age of 4 years and tends to diminish in severity in adulthood. The most common seizure types are partial complex, tonic-clonic, tonic, and myoclonic seizures. Although about $50 \%$ of seizures can be controlled by medication, intractable epilepsy occurs significantly more frequently in girls with obvious deceleration of head growth. The electroencephalogram is usually abnormal in RTT, but there is no clearly diagnostic pattern. Electroencephalogram patterns frequently 
seen in RTT include generalized slowing, monorhythmic theta waves, and focal and generalized spikes and sharp waves. In spite of this early and severe onset of epilepsy no negative effect on the long-term course and prognosis of RTT has been identified. Epilepsy tends to decrease after 20 years of age. Many girls become seizure-free or are well-controlled by medication [Steffenburg et al., 2001]. Status epilepticus does not occur more often than in severely mentally retarded children in general. Recent research reveals that the brain-derived neurotropic factor (BDNF) Met66 allele acts as a protective factor against seizures whereas missense mutations in the methyl-binding domain of MECP2 are more frequently associated with early seizures [Nectoux et al., 2008]. Brainstem events may be confused with seizures or are difficult to interpret as such by parents and caretakers. Signs of abnormal brainstem activity include blinking of the eyes, facial twitching, vacant spells without associated epileptiform activity and hypocapneic attacks with tetany and cyanosis. Classifying these clinical events requires simultaneous neurophysiological monitoring in real time of brainstem and cortical functions and correlation with behavior. Facial twitching with or without sudden changes in attention and eye deviation should not be a priori interpreted as epileptic paroxysms in a young child. This reflects more likely the ongoing process of immature brainstem activity and is not influenced by anti-epileptic drugs. Immature brainstem activity probably also accounts for the screaming spells, laughing spells, prolonged staring, and so on.

Autonomic Manifestations. Irregular breathing in the waking state associated with non-epileptic vacant spells is the most distressing feature in RTT, both for the girl and people around her [Smeets et al., 2006]. It reflects the immaturity of the brainstem and may contribute to sudden death. Low resting cardiac vagal tone and weak vagal response to hyperventilation and breath-holding suggest inadequate parasympathetic control. Neurophysiological studies have shown that these baseline brainstem functions are affected in RTT, while the baseline sympathetic tone remains at a neonatal level. Insight into brainstem physiology has introduced new terminology such as 'brainstem storm' and 'brainstem epilepsy' as physiological phenomena of abnormal spontaneous brainstem activation associated with altered breathing patterns [Julu et al., 2001]. Evaluating the brainstem functions in RTT requires detailed neurophysiology [Julu and Witt Engerström, 2005]. The primary pathophysiology is related to a defective control mechanism of carbon dioxide exhalation causing respiratory alkalosis or acidosis. Three car- diorespiratory phenotypes are described, each demanding a specific approach [Julu et al., 2008]: Forceful breathers usually have fixed low levels of $\mathrm{pCO}_{2}$ (chronic respiratory alkalosis); feeble breathers usually have fixed high levels of $\mathrm{pCO}_{2}$ (chronic respiratory acidosis) due to weak respiration, and physical activity during person-toperson contact can stimulate breathing but is short-lived; apneustic breathers accumulate carbon dioxide due to delayed and inadequate expirations.

Patients with RTT have a higher incidence of prolonged QT interval, and heart rate variability is diminished. These abnormalities likely result from impairment of autonomic nervous system control, reducing the electrical stability of the heart and precipitating sudden arrhythmia. Imbalance between preserved sympathetic tone and insufficient parasympathetic control is known to cause cardiac arrhythmia. Individuals with prolonged QT interval associated with abnormal breathing pattern are particularly at risk for cardiac arrhythmia, especially the forceful breathers. Of the deaths reported to the International Rett Syndrome Association in individuals less than 23 years of age, 22\% have been sudden, unexpected deaths, in comparison with $2.3 \%$ in the general population up to the same age [Kerr and Witt Engerström, 2001].

Cold Extremities. These are common in RTT due to poor perfusion as a consequence of altered autonomic control. Secondary vascular changes in the long term lead to abiotrophic changes in the lower limb and feet.

Agitation. This is seen as the consequence of the sympathovagal imbalance with unrestrained sympathetic activity in individuals with RTT. Some of them are abnormally prone to agitation and even 'sham rage' (personal experience).

Tooth Grinding. This is very common in RTT and has its characteristic fine cracking sound. It may lead to dental attrition. Botulinum toxin infiltration is used in extreme bruxism to relieve the muscle tone in the jaw with positive results on comfort and behavior [Laskawi, 2008].

Screaming Spells. Some teens and adults experience periodic sudden violent screaming that can last for hours and even for days. They often seem to signal extreme pain, though thorough examination does not reveal any somatic abnormality. This is not an epileptic phenomenon; it has been described as 'brain-pain-crying' [Smeets et al., 2003]. Others are abnormally prone to agitation and scream when they do not feel safe for whatever reason.

Sleep Abnormalities. They are more or less a constant feature of RTT. They include night laughter, prolonged wakefulness or early morning awakening. These prob- 
lems may persist into adult life. The mechanism behind this disruptive night awakening and daytime sleeping is not well-understood. It might be related to the autonomic dysfunction that has been attributed to midbrain and brainstem 'immaturity'.

Impaired Nociception. This is often seen in RTT and is sometimes confined to specific body parts. Rarely, the skin can present with easy bruising and blister formation, especially in severe classical RTT. These lesions are different from decubitus wounds. Impairment of pain perception probably represents delayed sensitivity to pain.

\section{RTT Variants and Differential Diagnosis}

An older girl or adult with classic RTT will be readily recognized clinically. In a young infant, however, the diagnosis can be difficult. Angelman syndrome is perhaps the most difficult clinical differential diagnosis as there is some degree of phenotypic overlap, particularly with regard to the severe developmental delay, motor impairment, stereotyped movements, absence of speech and epilepsy. The behavioral phenotype in Angelman syndrome [Williams et al., 2010] is a critical discriminator, though episodic laughter is not uncommon in RTT. Distinguishing features include a history of early regression in RTT, but this may not be obvious. Electroencephalographic features may be very helpful in the differential diagnosis.

Infantile neuronal ceroid lipofuscinosis (Batten disease) has a more rapidly progressive and degenerative course. It includes retinal involvement leading to blindness.

Disintegrative infantile psychosis is sometimes suggested as a diagnosis in RTT with predominantly autistic features in the early course of the syndrome. Characteristic RTT features may develop later. Autism in a girl without any dyspraxic or dystonic symptom is rarely seen in $\mathrm{MeCP}$ 2-related disorder. In doubtful cases resembling RTT or Angelman syndrome and where both disorders were excluded on a molecular basis, haploinsufficiency of TCF4 has been found [Zweier et al., 2007, 2008]. TCF4 is a gene encoding transcription factor 4 (TCF4) that was identified as the underlying cause of Pitt-Hopkins syndrome, an underdiagnosed mental-retardation syndrome associated with overbreathing and characteristic dysmorphism.

The variant or atypical RTT in MECP2-related disorder, in general, is referred to as 'forme fruste' based on a more protracted clinical course with more preserved communicative and motor abilities. Other variant forms are those with congenital onset, infantile seizure onset (Hanefeld variant), preserved speech (Zapella variant) and late regression depending on the age of onset or the principal feature dominating their clinical profile. Congenital RTT is rare and in most cases related to mutation in FOXG1. In the infantile seizure onset variant, hypsarrhythmia starting at the age of 2-4 months hides the features of the early stages of RTT. When the epileptic encephalopathy stabilizes and is controlled by medication, the girls regain a more or less clear RTT profile with some recovery of interaction. It is now considered as the Hanefeld variant and related to mutation in CDKL5 [Tao et al., 2004]. Preserved speech in RTT is not so rare and related to an overall better level of development. The late regression variant, with RTT starting at preschool or early school age, is also rare and very variable in clinical presentation starting with decline in school performance and total IQ.

The MECP2 duplication syndrome as seen in males can also be present in girls as a consequence of X/autosomal translocation. It may resemble RTT in the beginning, but the disorder is clinically more severe and different from classic RTT on the long term [Ramocki et al., 2010].

\section{MECP2-Related Disorders in Males}

For a long time, RTT was thought to be an X-linked dominant condition with lethality in hemizygous males. Since mutation in the MECP2 gene was found to be the cause of RTT, MECP2 mutations have also been reported in males. These males phenotypically have classical RTT when the mutation is present as somatic mosaic or when the individual has an X-polysomy. In all other cases, males with MECP2-related disorders show diverse phenotypes different from classical RTT. The spectrum ranges from severe congenital encephalopathy, to severe intellectual disability with various neurological symptoms, occasionally in association with psychiatric illness and macro-orchidism, to mild intellectual disability alone [Kerr and Witt Engerström, 2001; Leonard et al., 2001; Moog et al., 2003; Villard, 2007]. Boys with a duplication of the whole MECP2 gene (and sometimes contiguous genes) may show infantile hypotonia, recurrent respiratory infection, severe intellectual disability, absence of speech development, seizures and progressive spasticity. The recurrence of respiratory infections may be a criterion to distinguish these cases from other syndromes, as they occur in a context of normal growth.

\section{Prevalence and Prognosis}

The prevalence of the classic syndrome is estimated at $1 / 10,000$ females at an age of 32 years [Fehr et al., 2011]. 
Current understanding of the classical and variant forms suggests that the overall prevalence is probably higher. The annual death rate in classic RTT has been estimated at about 1.2\% in the UK [Kerr, 1992], and little change in survival has been found in the last 30 years [Freilinger et al., 2010]. Most of deaths clustered between the ages of 15 and 20 years with causes related to the disorder (wasted condition and poor health, pneumonia and epilepsy). Poor autonomic control is considered likely to play a significant role [Julu et al., 2001]. About 25\%, however, were unexpected and sudden deaths that occurred across the age range. In the general population up to the same age the death rate was 2.3\% [Kerr and Witt Engerström, 2001].

\section{Pathogenesis}

The pathophysiological mechanisms underlying RTT are yet to be clarified. In table 2 , the cardinal clinical features of RTT are shown in relation to pathology. Inactivating mutations of the $M E C P 2$ gene are thought to result in partial or complete loss of the ability to silence promoters of genes that are no longer or not needed in particular cell types [Gabellini et al., 2004], resulting in abnormalities in numerous neurotransmitter systems (including cholinergic, dopaminergic, glutaminergic, serotoninergic and GABAergic transmission) and trophic factors (including nerve growth factor and brain-derived neurotrophic factor). Immuno-reactive cy tometric studies have shown that the presence of $\mathrm{MeCP} 2$ in differentiated neurons is decreased in RTT. This deprivation of MeCP2 probably plays an important role in pathogenesis [LaSalle, 2001]. The pathology of RTT differs from other disorders with intellectual disability in that the pattern of dendritic changes in the brain is unique [Armstrong et al., 1998; Armstrong and Kinney, 2001]. Brain weight is reduced in girls with RTT but does not diminish with age. The defined cause of this arrest in brain development and how this results in altered neurophysiology is not yet wellunderstood. There is evident failure of dendritic arborization in specific sites of the brain, correlating with the cortical localization of some of the significant motor and behavioral symptoms. In relation to the peculiar movement disorder in RTT, the substantia nigra, basal ganglia, cerebellum and spinal cord have been found to show specific alterations. Various neurotransmitter systems have also been studied with varied and inconclusive results apart from the demonstration of monoaminergic dysfunction [Nomura et al., 1985; Nomura and Segawa, 1992; Armstrong, 2002].

\section{Clinical Managment}

Early intervention and comprehensive life-long management of RTT can significantly improve the health and longevity of affected individuals. Management is optimized by involvement of a multidisciplinary team consisting of many different medical and paramedical specialists and an individualized approach [Smeets and Schrander-Stumpel, 2010].

Strategies targeting specific symptoms as opposed to the full pathological picture may prove encouraging. There has been a special focus on anti-epileptic treatment [Nissenkorn et al., 2010]. A wealth of research has also been dedicated to improve autonomic nervous system function in patients with RTT. Three cardiorespiratory phenotypes are described, each demanding a specific approach [Julu et al., 2008]. There are also emerging data demonstrating sleep disorder and nighttime autonomic dysregulation [Weese-Mayer et al., 2006; Rohdin et al., 2007; Lioy et al., 2011].

Clinical management should essentially consist of rehabilitation programs adapted to the patients' individual needs, in which control of posture and movement and interaction in communication occupy a central place. Anxiety should be recognized and coping strategies widely vary, though a given child may often respond to the same strategy. Restraining hand movements has had variable results. Gentle framing (e.g. seating) may be effective to promote a shared activity. Children with RTT may show some interest in repetitive, dialog-like activities involving large objects (e.g. tapping on a large ball), or whole-body movements involving a postural challenge, such as sliding down an inclined plane. Repetitive activities seem more appropriate than variation with respect to patients' anxiety. Initiation of movement often needs to be facilitated by the therapist.

Habilitation programs will likely remain the cornerstone of management of individuals with RTT, although a better understanding of the molecular pathophysiology of these conditions is expected to lead to promising therapeutic approaches, including pharmacological agents interfering with neurotransmitter systems, gene expression or altering DNA methylation.

\section{Genetics}

\section{The MECP2 Gene}

$\mathrm{RTT}$ is a monogenic $\mathrm{X}$-linked dominant disorder due to mutations in MECP2 encoding the methyl-CpG-binding protein MeCP2 [Amir et al., 1999]. This gene, located in 
the Xq28 region, codes for the prototype of the family of proteins sharing a methyl-binding domain. Mutations in the coding region of this gene can be identified in approximately $80 \%$ of patients with the clinical diagnosis. Seven recurrent point mutations account for about $70 \%$ of the cases [Amir et al., 1999; Philippe et al., 2006]. Particularly prevalent are 4 missense mutations (i.e. R106W, R133C, T158M, R306C) and 4 nonsense protein-truncating mutations (i.e. R168X, R255X, R270X, R294X). There are about $10 \%$ of cases due to $\mathrm{C}$-terminal deletions and about $6 \%$ with complex chromosomal rearrangements [Chahrour and Zoghbi, 2007]. A small proportion of cases are due to mutations in a splicing isoform of MeCP2 (MECP2E1).

$M E C P 2$ contains a methyl-CpG-binding domain, a transcription repression domain, 2 nuclear localization signals, and a C-terminal segment. The coding sequence for the methyl-CpG-binding domain is split between exons 3 and 4, while the transcription repression domain lies entirely within exon 4 . The methyl-CpG-binding domain binds specifically to 5-methyl-cytosine throughout the genome. The transcription repression domain interacts with histone deacetylase and the transcription silencer co-repressor Sin3A. The nuclear localization signals mediate the transport of the $\mathrm{MeCP} 2$ protein into the nucleus, and the C-terminal segment facilitates binding to the nucleosome core [Chandler et al., 1999; Huppke et al., 2000]. These interactions result in deacetylation of histones and chromatin condensation, which leads to repression of transcription [Nan et al., 1998; Wan et al., 2001].

\section{$\mathrm{MeCP} 2$ Function}

The MeCP2 protein is ubiquitously present but particularly abundant in the brain [Amir et al., 1999]. It is thought to suppress the transcription of other tissue-specific genes whose activity is not required [Yntema et al., 2002]. Loss of function of MeCP2 in cells, especially in differentiated post-mitotic neurons, may lead to inappropriate over-expression of these other genes, with potentially damaging effect during central nervous system maturation [Ellaway and Christodoulou, 2001]. The knockout mouse, deficient in the Mecp2 gene, presents several features of RTT. The search for target genes that are derepressed in these mice has not yielded any clear candidates, except BDNF. Normal Mecp2 regulates the expression of this gene that is essential for learning and neural plasticity [Chen et al., 2003; Martinowich et al., 2003].

\section{Diagnostic Testing}

It is important to emphasize that in spite of the progress in molecular diagnostics, the diagnosis of RTT re- mains strongly based on clinical criteria. The availability of molecular testing may lead to early confirmation of a clinical suspicion of RTT in an infant girl. More than 200 different MECP2 mutations and several polymorphic variants have been described. They are listed in the database accessible through the International Rett Syndrome Association website (www.rettsyndrome.org). In individuals with classical RTT there is a more than $90 \%$ mutation detection rate using sequencing techniques to analyze the coding regions. With additional analysis using multiplex ligation-dependent probe amplification, the mutation detection rate rises to more than $95 \%$ in classical RTT. Gross rearrangements in the coding region (exons 3 and 4) were first established by Southern blot analysis in girls with severe classical RTT [Bourdon et al., 2001; Yaron et al., 2002; Schollen et al., 2003]. These gross rearrangements, consisting of larger deletions or duplications, are not detected by standard mutation screening techniques (denaturing high-performance liquid chromatography, long-range polymerase chain reaction and sequencing of the coding region). They are only detected by the multiplex ligation-dependent probe amplification technique that is now standard for initially mutationnegative females with RTT.

In variant RTT the frequency of detectable mutations in MECP2 is lower. However, when the clinical criteria for diagnosis of variant RTT are strictly followed a certain number of these cases will appear to be another, not MeCP2-related, disorder.

More than $95 \%$ of the MECP2 mutations occur de novo, and in the vast majority of affected individuals they occur on the paternal X chromosome [Girard et al., 2001]. Missense mutations seem to cluster in the methyl-CpGbinding domain, while nonsense or frame-shift mutations truncate the protein beyond this domain affecting the transcription repression domain and C-terminal segment of the gene.

\section{Genotype-Phenotype Correlation}

Correlation between the type and location of the mutation and the clinical severity of RTT is difficult and troublesome [Halbach et al., 2012]. Some have found more severe manifestations in children with nonsense mutations and milder expression in the late truncating mutations located at the deletion hot spot in the C-terminal segment [Huppke et al., 2000; Smeets et al., 2005, 2009; Bebbington et al., 2010]. The fact that the MECP2 gene is subject to $\mathrm{X}$ chromosome inactivation in females raises the question of the influence of this phenomenon over the phenotypic features [Zoghbi et al., 1990; Schanen 
et al., 1997]. In the rare familial cases of RTT a favorable skewed X chromosome inactivation has been found in phenotypically normal carrier mothers. In addition, affected twin sisters with different clinical severity have shown differential $\mathrm{X}$ inactivation patterns [Ishii et al., 2001]. These reports seem to argue in favor of an influence of X chromosome inactivation. But in general it cannot be used as a prognosticator in RTT [Archer et al., 2007]. Other hypotheses have been proposed to account for variations in features and clinical severity, including the presence of one or more modifier genes mediating the effects of MeCP2. For example, a 'digenic' model was suggested in which a 'mutated' allele in another gene leads to a protein with decreased function, thereby affecting the phenotypic outcome of the MECP2 gene mutation [Renieri et al., 2003].

\section{Genetic Counseling}

When a mutation is found in a child, the mother should be tested to see if she is an asymptomatic carrier. However, since more than 95\% of RTT mutations are de novo, the recurrence risk with a negative family history is very low (less than $0.1 \%$ ). Prenatal diagnosis can be offered to parents in subsequent pregnancies to evaluate the low likelihood of gonadal mosaicism. When the mother is an asymptomatic carrier, the recurrence risk is $50 \%$. This includes the risk of intra-uterine death or severe neonatal encephalopathy in a male.

\section{Animal Models}

The molecular characterization of RTT has allowed the development of animal models that can provide important insights into the pathophysiological mechanisms involved in various aspects of the syndrome. In particular, they may increase our knowledge of the processes underlying the evolution, development, maintenance, alteration, control or biological significance of the phenotypic manifestations. Comparative studies with wild-type animals or between different animal models contribute to the understanding of the correlation between genotype and phenotype. Animal models also enable investigators to test potential therapeutic approaches. However, the biochemical, anatomic and functional differences between animal models and humans must be born in mind, as some extrapolations may be difficult or impossible. These difficulties may occur at different levels from molecular biology to behavior. For example, it is difficult to know to what extent a feature such as cogni- tive impairment, which is central in RTT, is appropriately modeled by the eventual difficulties displayed by mice undergoing behavioral tests available for assessing mouse behavior attention, learning and memory. Although evidence suggests that some general cognitive processes are shared by mice and men, attempts to transpose the involved complexity from one species to the other would be hazardous, not to mention difficulties in comparing levels of competence. Some important aspects of cognitive functioning that are specifically relevant to RTT, such as absence of speech, cannot be approached in animals. Other behavioral aspects, such as hyperactivity, may arguably be equivalent in mice and men. Motor control might be a more promising area, as it seems to involve better defined and more conserved pathways. Neurophysiological approaches, with longstanding contributions to the understanding of human physiology and pathophysiology, may prove to be most useful. This would apply to many aspects of neural processing, epileptic activity and other electrophysiological activities, e.g. the brainstem events.

Mice are the most commonly used mammal species in animal research. This is due to several factors. There are remarkably physiological, anatomic and genetic similarities between mice and humans (more than $80 \%$ of DNA sequences appear to be shared). Other favorable factors include small size, short developmental course and reproductive cycle, relatively easy maintenance in captivity, mild behavior and other technical reasons relating to genetic manipulation. An important feature common to the currently available mouse models of RTT is the fact that they show phenotypic abnormalities that can be related to the human disease.

Although null mutation is not lethal in male mice, disturbances can be observed in knockout males from around 3-6 weeks of age and they die around 10 weeks of age. Mice with the truncating mutation Mecp2 308/ ${ }^{\mathrm{Y}}$ [Shahbazian et al., 2002] die around the age of 15 months, i.e. much later than conditional knockout mice.

$\mathrm{Mecp}^{+/-}$female mice, which are homologous to human patients with RTT, show no abnormalities when they are young. They are even fertile. However, they gradually show more inertia and hindlimb clasping beyond the age of 3 months, and later on they may show breathing irregularities.

There seems to be a clear effect of MeCP2 dosage. A 2 -fold increase in MeCP2 expression results in a progressive neurological disease with cognitive impairment, speech and social problems [Collins et al., 2004], reminiscent of the human condition associated with MECP2 du- 
plication [Van Esch et al., 2005]. Conversely, expressing only half of the MeCP2 protein is associated with altered social behavior [Samaco et al., 2008].

\section{Future Therapeutic Perspectives}

Given the function of $M E C P 2$ as a ubiquitous transcriptional repressor, a lot of studies have concentrated on identifying downstream genes to account for the phenotype of RTT. This approach has not been very successful as few significant findings have emerged if any. X-linked gene expression is not compromised, be it in patients with RTT or animal models based on MECP2 inactivation. The absence of generalized deregulation of gene expression may be due to functional redundancy between methyl-CpG-binding proteins. It must be noted, however, that alteration in target gene expression may be activity-dependent [Wang et al., 2006].

Another approach of research into RTT molecular pathophysiology concerns epigenetic influences. This may prove to have implications for developing therapeutic strategies. Patients with RTT show changes in global histone acetylation profiles [Shahbazian et al., 2002], which may be correlated to the severity of the mutation and the phenotype in peripheral cells [Kaufmann et al., 2005]. There have been contrasting results between patients and mouse models in terms of levels of $\mathrm{H} 3$ acetylation, and of $\mathrm{H} 3 \mathrm{~K} 9$ trimethylation.

Studies aiming at increasing the available pool of methyl groups through intermediate metabolites such as folic acid and betaine, which are known to be involved in the DNA methylation pathway, in the hope of increasing the degree of methylation of some $\mathrm{CpG}$ sites and leading to transcriptional repression either by other methylbinding proteins or by a mutant $\mathrm{MeCP} 2$ with altered affinity, have failed to demonstrate improvements in the clinical features of RTT [Glaze et al., 2009]. Recently, reevaluation of MeCP2 genomic binding sites has shown that only a small proportion $(6 \%)$ of these are located inside $\mathrm{CpG}$ islands, suggesting that the primary function of $\mathrm{MeCP} 2$ is not to bind methylated promoters for repressing transcription [Yasui et al., 2007]. Alternatively, the role of $\mathrm{MeCP} 2$ in shaping chromosomal architecture may be much more relevant [Clouaire and Stancheva, 2008]. In this perspective, $\mathrm{MeCP} 2$ could act by inducing chromatin reorganization during cellular differentiation [Brero et al., 2005]. Another approach would consist of targeting translational problems when MECP2 carries nonsense mutations which are associated with premature stop codons. Pharmacological agents such as aminoglycoside antibiotics (e.g. gentamycin) may enable ribosomal readthrough of such stop codons during translation by promoting insertion of another amino acid that causes a missense mutation [Martin et al., 1989]. This may potentially result in the production of a full-length functional protein with virtually preserved activity. The recent exponential research on induced pluripotent stem cells may prove to be a promising possibility for cell therapy with a potential of reorganizing neural circuitry [Gaspard and Vanderhaeghen, 2011].

There has been much hope of developing a targeted therapy since the widely publicized reversibility of abnormal features in mouse models. As RTT does not seem to be characterized by widespread neuronal death, the hypothesis that $\mathrm{MeCP} 2$ is required to stabilize and maintain the mature neuronal state has been reemphasized with a view to treatment. Resumed expression of $\mathrm{MeCP} 2$ was thus obtained in a mouse model with age-related expression. Activation of MeCP2 in null mutant mice resulted in normalization of the tests carried out previously, leading to a phenotype that was indistinguishable from that of wild-type controls [Guy et al., 2007]. Studies of Bdnf overexpression in the brain of Mecp2 mutant mice were based on the notion that $B D N F$ is a target gene of MeCP2 and deletion of $B d n f$ in $\mathrm{MeCp} 2$ mutants were associated with an earlier onset of Rett-like symptoms. Bdnf overexpression extended the life span, rescued a locomotor defect, and reversed an electrophysiological deficit observed in Mecp2 mutants [Chang et al., 2006]. Moreover, whereas reduced $B d n f$ expression in the brainstem correlated with respiratory dysfunction in Mecp2 mutant mice, enhanced $B d n f$ expression improved respiratory symptoms [Ogier et al., 2007]. In another approach aiming at optimizing deficient synaptic maturation, IGF1 treatment was shown to result in a partial rescue of a number of abnormalities seen in Mecp2 mutant mice, including life span, locomotor activity, respiratory function, brain weight, concentration of a postsynaptic density protein in motor cortex, spine density on motor cortex neurons, excitatory synaptic transmission in sensorimotor cortex neurons, and cortical circuit plasticity [Tropea et al., 2009]. This might open the way toward possible (partial) compensation for the virtual absence of $\mathrm{MeCP} 2$ where and when it is needed. However, confusion may arise in association with the use of terms such as 'cure' to characterize reversal of selected abnormalities in rescued genetically engineered animal models [Dan, 2009]. 'Cure' implies recovery from an illness, which is deceptive in this context. Brain development heavily relies on orderly pro- 
cesses that start in the embryo, drawing developmental trajectories. Although the issue of neuronal development has been poorly addressed in RTT, it is likely to be altered given documented impairment in neuronal functioning in patients and animal models. Diagnosis is always made relatively late in the brain developmental history: late infancy at best and later childhood in most cases. Furthermore, there are still major technical limitations to application of similar strategies in human patients. It must also be borne in mind that unpredictable alteration in $M E C P 2$ expression (related to random X chromosome inactivation) may be hazardous as discussed above. This remark is probably less important if basal MECP2 level of expression is very low. Transgene complementation in female mice with low Mecp2 expression resulted in partial behavioral improvement [Jugloff et al., 2008].

Considerable progress is being made in understanding the mechanisms of autonomic cardiorespiratory dysfunction in RTT [Julu et al., 2008]. While respiration has received more attention in animal studies [Katz et al., 2009] compared to cardiovascular function, there is a strong suggestion that insufficient GABA synaptic transmission may be a common thread that impairs the 2 neuronal systems [Lioy et al., 2011].

\section{References}

- Amir RE, Van den Veyver IB, Wan M, Tran CQ, Francke U, Zoghbi HY: Rett syndrome is caused by mutations in X-linked MECP2, encoding methyl-CpG-binding protein 2. Nat Genet 23:185-188 (1999).

-Archer H, Evans J, Leonard H, Colvin L, Ravine $\mathrm{D}$, et al: Correlation between clinical severity in patients with Rett syndrome with a p.R168X or p.T158M MECP2 mutation, and the direction and degree of skewing of $\mathrm{X}$ chromosome inactivation. J Med Genet 44: 148-52 (2007).

Armstrong DD: Neuropathology of Rett syndrome. MRDD Research Reviews 8:72-76 (2002).

Armstrong DD, Kinney HC: The neuropathology of the Rett disorder, in Kerr A, WittEngerström I (eds): Rett Disorder and the Developing Brain. The Neuropathology of the Rett Disorder, pp 57-84 (Oxford University Press, Oxford 2001).

-Armstrong DD, Dunn JK, Antalffy B: Decreased dendritic branching in frontal, motor, limbic cortex in Rett syndrome compared with trisomy 21. J Neuropathol Exp Neurol 57:10131017 (1998).

- Bebbington A, Percy A, Christodoulou J, Ravine D, Ho G, et al: Updating the profile of C-terminal MECP2 deletions in Rett syndrome. J Med Genet 47:242-248 (2010).

- Bourdon V, Philippe C, Labrune O, Amsallem D, Arnould C, Jonveaux P: A detailed analysis of the MECP2 gene: prevalence of recurrent mutations and gross rearrangements in Rett syndrome patients. Hum Genet 108:43-50 (2001).

- Brero A, Easwaran HP, Nowak D, Grunewald I, Cremer T, et al: Methyl CpG-binding proteins induce large-scale chromatin reorganization during terminal differentiation. J Cell Biol 169:733-743 (2005).

-Chahrour M, Zoghbi HY: The story of Rett syndrome: from clinic to neurobiology. Neuron 56:422-437 (2007).
Chandler SP, Guschin D, Landsberger N, Wolffe AP: The methyl-CpG-binding transcriptional repressor MeCP2 stably associates with nucleosomal DNA. Biochemistry 38: 7008-7018 (1999).

Chang Q, Khare G, Dani V, Nelson S, Jaenisch R: The disease progression of Mecp2 mutant mice is affected by the level of BDNF expression. Neuron 2006, 49, 341-348.

Chen W, Chang Q, Lin Y, Meissner A, West A, et al: Derepression of BDNF transcription involves calcium-dependent phosphorylation of MECP2. Science 302:885 (2003).

Clouaire T, Stancheva I: Methyl-CpG binding proteins: specialized transcriptional repressors or structural components of chromatin? Cell Mol Life Sci 65:1509-1522 (2008).

Collins AL, Levenson JM, Vilaythong AP, Richman R, Armstrong DL, et al: Mild overexpression of $\mathrm{MeCP} 2$ causes a progressive neurological disorder in mice. Hum Mol Genet 13:2679-2689 (2004).

Dan B: Angelman syndrome: current understanding and research prospects. Epilepsia 50:2331-2339 (2009).

Dan B, Cheron B: Postural control in children with Rett syndrome or Angelman syndrome; in Hadders-Algra M, Brogren Carlberg E (eds): Posture: A Key Issue in Developmental Disorders, pp 148-169 (Mac Keith Press, London 2008).

Elian M, de M Rudolf N: Observations on hand movements in Rett syndrome: a pilot study. Acta Neurol Scand 94:212-214 (1996).

Ellaway C, Christodoulou J: Rett syndrome: clinical characteristics and recent genetic advances. Disabil Rehabil 23:98-106 (2001).

Fehr S, Bebbington A, Nassar N, Downs J, Ronen GM, et al: Trends in the diagnosis of Rett syndrome in Australia. Pediatr Res 70:313319 (2011).
Freilinger M, Bebbington A, Lanator I, De Klerk N, Dunkler D, et al: Survival with Rett syndrome: comparing Rett's original sample with data from the Australian Rett Syndrome Database. Dev Med Child Neurol 52: 962-965 (2010).

Gabellini D, Green MR, Tupler R: When enough is enough: genetic diseases associated with transcriptional derepression. Curr Opin Genet Dev 14:301-307 (2004).

Gaspard N, Vanderhaeghen P: From stem cells to neural networks: recent advances and perspectives for neurodevelopmental disorders. Dev Med Child Neurol 53:13-17 (2011).

- Girard M, Couvert P, Carrie A, Tardieu M, Chelly J, et al: Parental origin of de novo MECP2 mutations in Rett syndrome. Eur J Hum Genet 9:231-236 (2001).

-Glaze DG, Percy AK, Motil KJ, Lane JB, Isaacs JS, et al: A study of the treatment of Rett syndrome with folate and betaine. J Child Neurol 24:551-556 (2009).

Guy J, Gan J, Selfridge J, Cobb S, Bird A: Reversal of neurological defects in a mouse model of Rett syndrome. Science 315:1143-1147 (2007).

Hagberg B, Gillberg C: Rett variants-Rettoid phenotypes; in Hagberg B (ed): Rett Syndrome, Clinical and Biological Aspects, Clinics in Developmental Medicine vol. 127, pp 40-60 (MacKeith Press/Cambridge University Press, Cambridge 1993).

-Hagberg B, Aicardi J, Dias K, Ramos O: A progressive syndrome of autism, dementia, ataxia and loss of purposeful handuse in girls: Rett's syndrome: report of 35 cases. Ann Neurol 14:471-479 (1983).

Hagberg B, Stenbom Y, Engerström IW: Head growth in Rett syndrome. Brain Dev 23 (suppl 1):S227-S229 (2001)

-Hagberg B, Hanefeld F, Percy A, Skjeldal O: An update on clinically applicable diagnostic criteria in Rett syndrome. Eur J Peadiatr Neurol 6:293-297 (2002). 
- Halbach N, Smeets E, van den Braak N, van Roozendaal K, Blok R, et al: Genotype-phenotype relationships as prognosticators in Rett syndrome should be handled with care in clinical practice. Am J Med Genet A 158A:340-350 (2012).

-Huppke P, Laccone F, Kramer N, Engel W, Hanefeld F: Rett syndrome: analysis of MECP2 and clinical characterization of 31 patients. Hum Mol Genet 9:1369-1375 (2000).

-Ishii T, Makita Y, Ogawa A, Amamiya S, Yamamoto $\mathrm{M}$, et al: The role of different $\mathrm{X}$-inactivation pattern on the variable clinical phenotype with Rett syndrome. Brain Dev 23 (suppl 1):S161-S164 (2001).

Ishikawa A, Goto T, Narasaki M, Yokochi K, Kitahara H, Fukuyama Y: A new syndrome of progressive psychomotor retardation with peculiar stereotyped movements and autistic tendency: a report of three cases. Brain Dev 3:258 (1978).

-Jugloff DG, Vandamme K, Logan R Visanji NP, Brotchie JM, Eubanks JH: Targeted delivery of a Mecp2 transgene to forebrain neurons improves the behavior of female Mecp2-deficient mice. Hum Mol Genet 17:1386-1396 (2008).

-Julu PO, Witt Engerström I: Assessment of the maturity-related brainstem functions reveals the heterogeneous phenotypes and facilitates clinical management of Rett syndrome. Brain Dev 27 (suppl 1):S43-S53 (2005).

-Julu PO, Kerr AM, Apartopoulos F, Al-Rawas S, Witt-Engerström I, et al: Characterisation of breathing and associated central autonomic dysfunction in the Rett disorder. Arch Dis Child 85:29-37 (2001).

-Julu PO, Witt Engerström I, Hansen S, Apartopoulos F, Witt B, et al: Clinical update addressing the cardiorespiratory challenges in medicine posed by Rett syndrome: The Frösö Declaration. Lancet 371:1981-1983 (2008).

Katz DM, Dutschmann M, Ramirez JM, Hilaire G: Breathing disorders in Rett syndrome: progressive neurochemical dysfunction in the respiratory network after birth. Respir Physiol Neurobiol 168:101-108 (2009).

Kaufmann WE, Jarrar MH, Wang JS, Lee YJ, Reddy S, et al: Histone modifications in Rett syndrome lymphocytes: a preliminary evaluation. Brain Dev 27:331-339 (2005).

Kerr AM: Rett syndrome British longitudinal study (1982-1990) and 1990 survey; in Roosendaal JJ (ed): Mental Retardation and Medical Care, pp 143-145 (Kerckbosch Publisher, Zeist 1992).

Kerr A, Witt Engerström I: Rett Disorder and the Developing Brain (Oxford University Press, Oxford 2001).

LaSalle JM, Goldstine J, Balmer D, Greco CM: Quantitative localization of heterogeneous methyl-CpG-binding protein 2 (MeCP2) expression phenotypes in normal and Rett syndrome brain by laser scanning cytometry. Hum Mol Genet 10:1729-1740 (2001).
Laskawi R: The use of botulinum toxin in head and face medicine: an interdisciplinary field. Head Face Med 10:4-5 (2008).

Leonard H, Silberstein J, Falk R, Houwink-Manville I, Ellaway C, et al: Occurrence of Rett syndrome in boys. J Child Neurol 16:333338 (2001).

Lioy DT, Wu WW, Bissonnette JM: Autonomic dysfunction with mutations in the gene that encodes methyl-CpG-binding protein 2: insights into Rett syndrome. Auton Neurosci 161:55-62 (2011)

-Martin R, Mogg AE, Heywood LA, Nitschke L, Burke JF: Aminoglycoside suppression at UAG, UAA and UGA codons in Escherichia coli and human tissue culture cells. Mol Gen Genet 217:411-418 (1989).

Martinowich K, Hattori D, Wu H, Fouse S, He F, et al: DNA methylation-related chromatin remodeling in activity-dependent $\mathrm{BDNF}$ gene regulation. Science 302:890 (2003).

Moog U, Smeets E, van Roozendaal K, Schoenmakers S, Herbergs J, et al: Neurodevelopmental disorders in males related to the gene causing Rett syndrome in females (MECP2). Eur J Paediatr Neurol 7:5-12 (2003).

Motil KJ, Schultz RJ, Browning K, Trautwein L, Glaze DG: Oropharyngeal dysfunction and gastroesophageal dysmotility are present in girls and women with Rett syndrome. J Pediatr Gastroenterol Nutr 29:31-37 (1999).

-Nan X, Ng HH, Johnson CA, Laherty CD, Turner BM, et al: Transcriptional repression by the methyl-CpG-binding protein $\mathrm{MeCP} 2$ involves a histone deacetylase complex. Nature 393:386-389 (1998).

Nectoux J, Bahi-Buisson N, Guellec I, Coste J, De Roux N, et al: The p.Val66Met polymorphism in the BDNF gene protects against early seizures in Rett syndrome. Neurology 70(22 Pt 2):2145-2151 (2008).

Neul JL, Kaufmann WE, Glaze DG, Christodoulou J, Clarke AJ, et al: Rett syndrome: revised diagnostic criteria and nomenclature. Ann Neurol 68:944-950 (2010).

Nissenkorn A, Gak E, Vecksler M, Reznik H, Menascu S, Ben Zeev B: Epilepsy in Rett syndrome - the experience of a National Rett Center. Epilepsia 51:1252-1258 (2010).

Nomura Y, Segawa M: Motor symptoms of the Rett syndrome: abnormal muscle tone, posture, locomotion and stereotyped movement. Brain Dev 14 (suppl):S21-S28 (1992).

Nomura Y, Segawa M, Higurashi M: Rett syndrome - an early catecholamine and indolamine deficient disorder? Brain Dev 7:334341 (1985).

Ogier M, Wang H, Hong E, Wang Q, Greenberg ME, Katz DM: Brain-derived neurotrophic factor expression and respiratory function improve after ampakine treatment in a mouse model of Rett syndrome. J Neurosci 27:10912-10917 (2007).

Pelc K, Dan B: Postural cortical myoclonus during gait in Rett syndrome. Epilepsy Behav 16: 188 (2009).
Philippe C, Villard L, De Roux N, Raynaud M, Bonnefond JP, et al: Spectrum and distribution of MECP2 mutations in 424 Rett syndrome patients: a molecular update. Eur J Med Genet 49:9-18 (2006).

Ramocki M, Tavyev Y, Peters S: The MECP2 duplication syndrome. Am J Med Genet A 152: 1079-1088 (2010).

Renieri A, Meloni I, Longo I, Ariani F, Mari F, et al: Rett syndrome: the complex nature of a monogenic disease. J Mol Med 81:346-354 (2003).

Rett A: Über ein eigenartiges hirnatrophisches Syndrom bei Hyperammonämie im Kindesalter. Wien Med Wochenschr 116:723728 (1966).

Rett A: Cerebral Atrophy associated with hyperammonaemia, in Vinklen PJ, Bruyn GW (eds): Handbook of Clinical Neurology, vol. 29, pp 305-329 (North Holland publishing company, Amsterdam 1977).

Rohdin M, Fernell E, Eriksson M, Albåge M, Lagercrantz H, Katz-Salamon M: Disturbances in cardiorespiratory function during day and night in Rett syndrome. Pediatr Neurol 37:338-344 (2007)

Samaco RC, Fryer JD, Ren J, Fyffe S, Chao HT, et al: A partial loss of function allele of methylCpG-binding protein predicts a human neurodevelopmental syndrome. Hum Mol Genet 17:1718-1727 (2008).

Schanen NC, Dahle EJ, Capozzoli F, Holm VA, Zoghbi HY, Francke U: A new Rett syndrome family consistent with X-linked inheritance expands the $\mathrm{X}$ chromosome exclusion map. Am J Hum Genet 61:634-641 (1997).

Schollen E, Smeets E, Deflem E, Fryns JP, Matthijs G: Gross rearrangements in the $M E C P 2$ gene in three patients with Rett syndrome: implications for routine diagnosis of Rett syndrome. Hum Mut 22:116-120 (2003).

Schultz R, Glaze D, Motil K, Hebert D, Percy A: Hand and foot growth failure in Rett syndrome. J Child Neurol 13:71-74 (1998).

-Shahbazian M, Young J, Yuva-Paylor L, Spencer C, Antalffy B, et al: Mice with truncated MeCP2 recapitulate many Rett syndrome features and display hyperacetylation of histone H3. Neuron 35:243-254 (2002).

Smeets EE, Schrander-Stumpel CT: Rett Syndrome, in Cassidy S, Allanson J (eds): Management of Genetic Syndromes, 3rd ed, pp 677-691 (John Wiley \& Sons Inc., Hoboken, NJ 2010).

Smeets E, Schollen E, Moog U, Matthijs G, Herbergs J, et al: Rett Syndrome in adolescent and adult females: clinical and molecular genetic findings. Am J Med Gen 122A:227-233 (2003).

-Smeets E, Terhal P, Casaer P, Peters A, Midro A, et al: Rett syndrome in females with CTS hot spot deletions: a disorder profile. Am J Med Genet A 132A:117-120 (2005).

-Smeets E, Julu P, van Waardenburg D, Engerström I, Hansen S, et al: Management of a severe forceful breather with Rett syndrome using carbogen. Brain Dev 28:625-632 (2006). 
Smeets EE, Chenault M, Curfs LM, SchranderStumpel CT, Frijns JP: Rett syndrome and long-term disorder profile. Am J Med Genet A 149A:199-205 (2009).

-Steffenburg U, Hagberg G, Hagberg B: Epilepsy in a representative series of Rett syndrome. Acta Paediatr 90:34-39 (2001).

Tao J, Van Esch H, Hagedorn-Greiwe M, Hoffmann K, Moser B, et al: Mutations in the $\mathrm{X}$-linked cyclin-dependent kinase-like 5 (CDKL5/STK9) gene are associated with severe neurodevelopmental retardation. Am J Hum Genet 75:1149-1154 (2004).

- Tropea D, Giacometti E, Wilson NR, Beard C, McCurry C, et al: Partial reversal of Rett syndrome-like symptoms in MeCP2 mutant mice. Proc Natl Acad Sci USA 106:20292034 (2009).

-Van Esch H, Bauters M, Ignatius J, Jansen M, Raynaud M, et al: Duplication of the MECP2 region is a frequent cause of severe mental retardation and progressive neurological symptoms in males. Am J Hum Genet 77: 442-453 (2005).

-Villard J: MECP2 mutations in males. J Med Genet 44:417-423 (2007).

Wan M, Zhao K, Francke U: MECP2 truncating mutations cause histone $\mathrm{H} 4$ hyperacetylation in Rett syndrome. Hum Mol Genet 10: 1085-1092 (2001).
Wang H, Chan SA, Ogier M, Hellard D, Wang Q, et al: Dysregulation of brain derived neurotrophic factor expression and neurosecretory function in Mecp2 null mice. J Neurosci 26:10911-10915 (2006).

-Weese-Mayer DE, Lieske SP, Boothby CM, Kenny AS, Bennett HL, et al: Autonomic nervous system dysregulation: breathing and heart rate perturbation during wakefulness in young girls with Rett syndrome. Pediatr Res 60:443-449 (2006).

Williams CA, Driscoll DJ, Dagli AI: Clinical and genetic aspects of Angelman syndrome. Genet Med 12:385-395 (2010).

Witt Engerström I: Rett Syndrome in Sweden. Neurodevelopment - disability - pathophysiology. Acta Paediatr Scand Suppl 369:1-60 (1990).

Yaron Y, Ben Zeev B, Shomrat R, Bercovich D, Naiman T, Orr-Urtreger A: MECP2 mutations in Israel: implications for molecular analysis, genetic counseling and prenatal diagnosis in Rett syndrome. Hum Mutat 20: 323-324 (2002).

Yasui DH, Peddada S, Bieda MC, Vallero RO, Hogart A, et al: Integrated epigenomic analyses of neuronal MeCP2 reveal a role for long-range interaction with active genes. Proc Natl Acad Sci USA 104:19416-19321 (2007).
Yntema HG, Oudakker AR, Kleefstra T, Hamel $B C$, van Bokhoven $\mathrm{H}$, et al: In-frame deletion in MECP2 causes mild nonspecific mental retardation. Am J Med Genet 107:81-83 (2002).

Zoghbi HY, Percy AK, Schulz RJ, Fill C: Patterns of $\mathrm{X}$ chromosome inactivation in the Rett syndrome. Brain Dev 12:131-135 (1990).

Zweier C, Peippo MM, Hoyer J, Sousa S, Bottani A, et al: Haploinsufficiency of TCF4 causes syndromal mental retardation with intermittent hyperventilation (Pitt-Hopkins syndrome). Am J Hum Genet 80:994-1001 (2007)

Zweier C, Sticht H, Bijlsma EK, Clayton-Smith J, Boonen SE, et al: Further delineation of PittHopkins syndrome: phenotypic and genotypic description of 16 novel patients. J Med Genet 45:738-744 (2008).

Zweier C, de Jong EK, Zweier M, Orrico A, Ousager LB, et al: CNTNAP2 and NRXN1 are mutated in autosomal-recessive Pitt-Hopkins-like mental retardation and determine the level of a common synaptic protein in Drosophila. Am J Hum Genet 85:655-666 (2009). 